\title{
Improvement of the bearing structure of the wagon-platform of the articulated type to ensure the reliability of the fixing on the deck of the railway ferry
}

\author{
Alyona Lovskaya ${ }^{1}$, Juraj Gerlici ${ }^{2}$, Oleksij Fomin $^{3}$, Kateryna Kravchenko, ${ }^{2,}$, Pavlo \\ Prokopenko ${ }^{4}$, and Tomas Lack $^{2}$ \\ ${ }^{1}$ Ukrainian State University of Railway Transport, Department of Wagons, 61050, Feuerbach Square 7, \\ Kharkov, Ukraine \\ ${ }^{2}$ University of Zilina, Department of transport and handling machines, 01026, Univerzitná 8215/1, \\ Zilina, Slovak Republic \\ ${ }^{3}$ State University of Infrastructure and Technology, Department of Cars and Carriage Facilities, 04071, \\ Kyrylivska str., 9, Kyiv, Ukraine \\ ${ }^{4}$ Branch "Research and design and technological Institute of Railway Transport \\ "PJSC”Ukrzaliznytsya", 03038, Ivana Fedorova, 39, Kyiv, Ukraine
}

\begin{abstract}
In the article the results of studies of loading of the bearing structure of a wagon-platform of an articulated type, created on the basis of the existing construction, during transportation on the railway ferry by sea are given. In order to ensure the reliability of the fixing of the bearing structure of the wagon-platform of the articulated type relative to the deck of the railway ferry, it is proposed to equip it with knots for fixing the chain ties. To study the dynamic loading of a wagon-platform of an articulated type during transportation on a railway ferry by sea, constructed a mathematical model of oscillations with angular displacements relative to the longitudinal axis of the vessel (loll/heel). The maximal values of accelerations, as components of dynamic load, acting on the bearing structure of a wagon-platform are determined. The obtained acceleration values are taken into account when calculating the strength of the bearing structure using the finite element method implemented in the CosmosWorks software environment. It is established that the maximum equivalent tension do not exceed permissible values. The carried out researches will promote increase of efficiency of combined transportations in the direction of the international transport corridors.
\end{abstract}

Keywords: wagon-platform, acceleration, dynamics, tension, strength, rail and ferry transportation

${ }^{*}$ Corresponding author: kkatherina@ukr.net

Reviewers: Radim Halama, Konrad Waluś 


\section{Introduction}

The development of a competitive environment in the railway market and increasing the efficiency of its operation, requires the commissioning of the rolling stock of the new generation, and combined transport systems. One of the most promising among such systems is the rail-ferry transportation, which has been successfully operating in Ukraine since 1954, on the example of the first railway-ferry route through the Kerch Strait between Taman and Kerch. In recent years, the geography of Ukraine's ferry connection with other countries has considerably expanded. One of the last among such routes is the chain of the international transport corridor, which connected the countries of Europe and Asia and began to operate from the beginning of last year. The railway ferry which serving this route is equipped with 13 railway tracks for the installation of wagons (five tracks on the upper and the main (middle) decks, three on the hold (bottom)) and can take on the board to 108 wagons with a size 1-T, 0-T, 01-T. For the wagon delivery to the upper and bilge decks, the ferry is equipped with a hydraulic lift. For horizontally moving of wagons between the tracks of the upper and bilge decks, there are turning sections with platforms. In order to ensure the stability of wagons during transportation by railway ferry in the conditions of the sea pitching, their fixing relative to the decks is carried out with the help of a complex of multiturning fixing devices: chain ties, mechanical stop-jacks, dead-end stoppers, brake shoes. Taking into account the accelerated pace of Ukraine's integration into the system of international transport corridors, it is expected to open new railway and ferry routes that will connect Ukraine with other states through the Black Sea and the Azov Seawater areas.

Transportation of a flat wagon on the railway ferry is accompanied by the action on their bearing structures of significant amounts of effort whose numerical values exceed those that work during operating relative to rails track. This may cause damage to the structural elements of the wagons, for which they are fixed relative to the decks, loss of stability and traffic safety violation of the railway ferry. Therefore, it is necessary to improve the bearing structures of flat wagon to ensure the reliability of their fixing relative to the decks and to take into consideration at the design stage the updated values of the dynamic loads acting on them when transported on the railway ferry.

\section{Analysis of recent researches}

The issue of designing a rolling stock of a new generation with improved technical and economic performance is given in [1-4].

Studies on the creation of ideal carrier structures of freight wagon bodies and the requirements that they must meet at the present stage of the development of rail transport are considered in $[5,6]$.

The study of the dynamic loading of a flat wagon, as one of the most common types of wagons in combined transportations, is given in [7, 8].

The work presents mathematical models that allow to determine the dynamic and strength characteristics of bearing structures of flat wagon at operating load conditions.

It is important to note that in the considered works there is no research on the possibility of exploiting the proposed designs of wagons in international rail-water communication, and the study of the strength of bearing structures of wagons at interaction with multi-turning means of railway ferry fixing.

The purpose of the article is improvement of the bearing structure of a flat wagon of an articulated type to ensure the reliability of fixing on the deck the railway ferry. 


\section{Presentation of the main material}

The insufficient level of replenishment of the Ukrzaliznytsia wagon park in recent years causes necessity for putting into operation new technical solutions to improve the existing bearing structures of wagon bodies to increase the function efficiency of railway transport. Therefore, it is proposed to create a flat wagon of an articulated type based on model 13-401 [9]. For the purpose of exploitation a flat wagon of an articulated type in international rail-water connection it is proposed to install knots on its bearing structure to fix a chain binder [10].

Placement of the fixing knots proposed to be carried out on the bolster beams of the flat wagon, which will allow providing spatial placement of the chain binder and the correspondence of the angles of its inclination in the space of normative documents [11]. From the side of bearing zones on the middle bogie, placement of nodes is carried out on the superstructures of the box-shaped intersection. The geometric parameters of the superstructures are determined on the basis of the conditions of their durability at loading through the nodes of fixing the chain binder and the geometry of the longitudinal beam of the frame on which they are placed.

The geometric parameters of the node were designed taking into account the sizes of the bolster beam of the wagon and hook of the chain binder.

On the basis of calculations, the received variant of the fixing knot construction shown in Fig. 1.

Direction 1 completely repeats the geometry of the hook coupler contour of the chain binder and is designed to interact with the fixing node. In order to reduce the concentration of tension in the zone of interaction of the node with the support part, a radial tide 2 is provided. The intermediation of the cylindrical part 3, the height of which was designed in view of the width of the hook by the clutch contour, provides a clear interaction between the hook and the knot. The intermediation of the cylindrical part 3, the height of which was designed in view of the width of the hook by the clutch contour, provides a clear interaction between the hook and the knot. Prismatic part 4 is designed to combine the working part of the node with the auxiliary, which consists of an amplification 5. The supporting parts 6 of the knot are intended for fixing it on the bolster beam of the wagon. The weight of the knot is about $10 \mathrm{~kg}$.

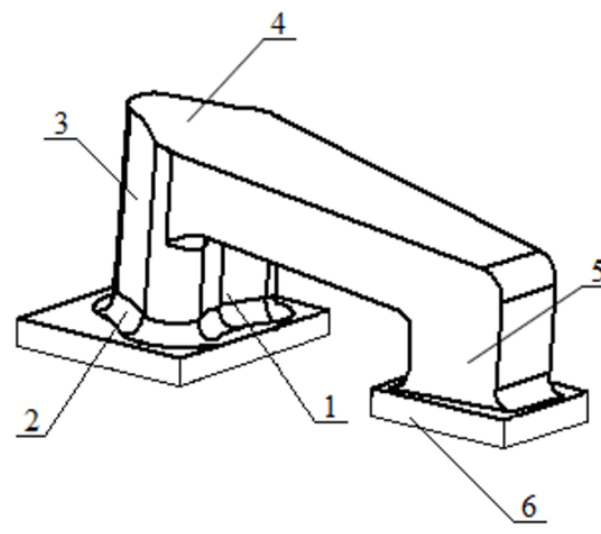

a)

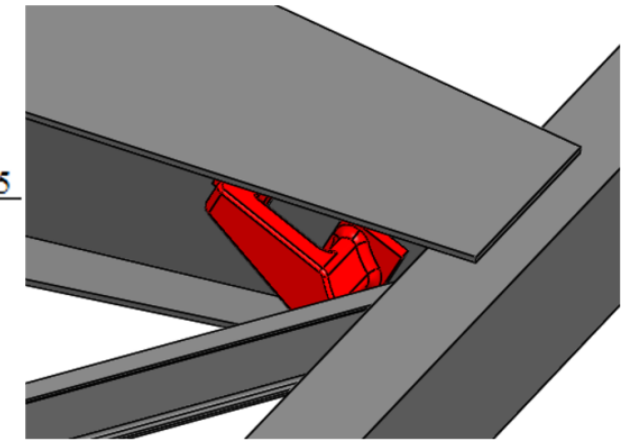

b)

Fig. 1. Node for fixing the hook of the chain binder: a) structural features; b) placement on a flat-wagon

For the study of the strength of the site for fixing the hook of the chain binder on the wagon, a calculation was made using the finite element method, the number of elements of 
the grid is determined by the graph-analytical method and amounted to 8531 , nodes -13829 . The calculation scheme of the node and its tense state are shown in Fig. 2.

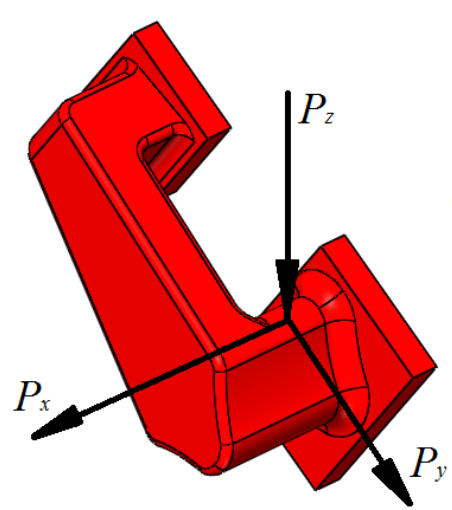

a)

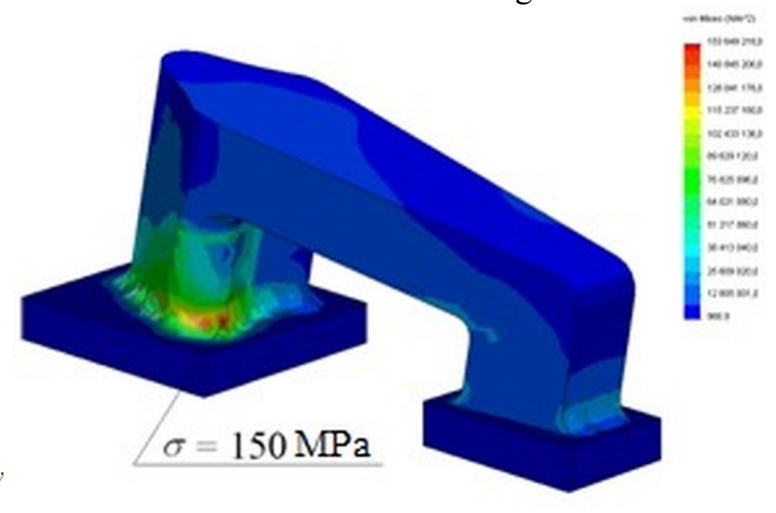

b)

Fig. 2. Calculation of the strength of the knot for fixing the hook of the chain binder: a) calculation scheme; b) a tense state

While strength analysis of element shown on Fig. 2, the load from hook was applied as equally distributed on the contact surface, element was rigidly grounded on his ends. The numerical value of the load is taken to be equal to $196.2 \mathrm{kN}(20 \mathrm{t})$, which corresponds to the maximum allowable load on which the chain binder is designed. Since the chain binder has a spatial arrangement, the forces acting on the fixing node from it were divided into three components.

On the basis of the calculations made, it is concluded that during interaction of the hook of the chain binder with the fixing node, the maximum equivalent tensions can be traced in the radial tidal zone, which binds the working part with the bearing, and have a value of about $150 \mathrm{MPa}$ which is lower than the permissible for the given steel grades (09G2D and 09G2C), in the transition and auxiliary parts of the tension have a small value $-10 \mathrm{MPa}$. The maximum displacement in the node is $0.02 \mathrm{~mm}$, the deformation is $6.2 \cdot 10^{-4}$ and is concentrated in the zone of radial tidal. The safe load factor of the construction is 2 .

That is, in case of force loading of the knot for fixing the hook of the chain binder, its strength is provided.

With the aim to study of fatigue strength for proposed connection, calculation in the Cosmos Works environment was taken out, $10^{7}$ cycles was investigated. Fatigue curve is obtained from dividing each value of stress of SN-curve by elastic module of ASME etalon material, than this obtained value is multiplied by the value of elastic module of used material [12] (Fig. 3). Fatigue study of proposed connection is based on pre-processed calculation of static strength with subsequently taken into account the cyclic load. According performed calculations, it can be concluded, that strength of proposed connection for specified load cycle number is guaranteed.

For the research of the strength of the welding seam under the applied force load calculation was made. In this case, the strength condition looks like:

$$
\sigma_{S}=\frac{3 \cdot M}{\left(\beta \cdot h_{S}\right) \cdot l_{S}^{2}}+\frac{N}{F} \leq R_{Y}^{C R},
$$

where $M$ - bending moment acting at the intersection of the seam; $\beta \cdot h_{s}$ - estimated seam thickness; $\beta$-coefficient of depth of the seam; $l_{s}$ - estimated seam length; $N$ - the calculated force acting on the connection; $F$ - area of connection; $R_{Y}^{C R}$ - calculated resistance of the seam. 


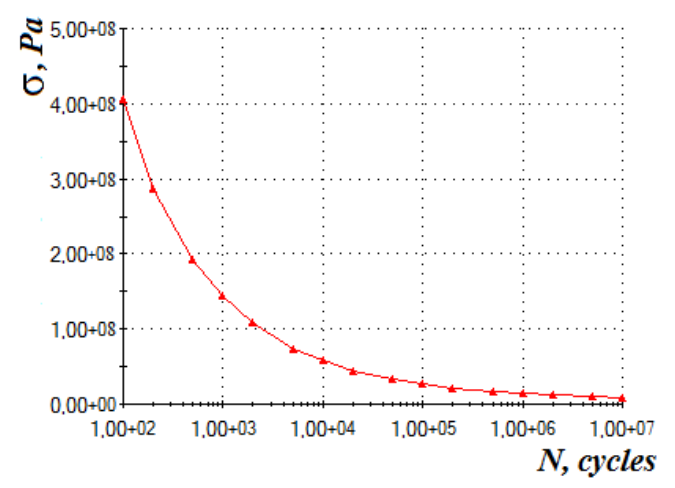

Fig. 3.The fatigue curve of metal structure of the knot for fixing the hook of the chain binder. Fixing the knot on the bolster beams of the wagon is carried out by welding

Based on the calculations received the value $\sigma_{s}=73 \mathrm{MPa}$ at $R_{Y}^{C R}=196 \mathrm{MPa}$. That is, the condition of strength is fulfilled.

To determine the dynamic loads that act on the supporting structure of a flat wagon of an articulated type with containers placed on it when transported on a railway ferry, a mathematical model is developed that takes into account the angular displacements of the system elements ("the railway ferry - the flat wagon-container") around longitudinal axis (roll/list), as the case of the greatest loading of wagon bearing structure during transportation by a railway ferry, also ensuring its stability relative to the deck (Fig. 4).

It is taken into account that the list is caused by the static effect of the wind on the surface projection of a railway ferry with flat wagons loaded with containers and located on the upper deck. In this case, the numerical value of the angle of the roll was about $12^{\circ}$.

$$
\left\{\begin{array}{l}
\left(\frac{D}{12 g}\left(B^{2}+4 z_{g}^{2}\right)\right) \ddot{q}_{1}+\left(\Lambda_{\theta} \cdot \frac{B}{2}\right) \dot{q}_{1}=p_{r f}^{\prime} \cdot \frac{h}{2}+\Lambda_{\theta} \cdot \frac{B}{2} \cdot F(t), \\
I_{f w}^{\theta} \cdot \ddot{q}_{2}=p_{f w}^{\prime} \cdot \frac{h_{f w}}{2}+M_{f w}^{D}+M_{f w}^{C}, \\
I_{c}^{\theta} \cdot \ddot{q}_{3}=p_{c}^{\prime} \cdot \frac{h_{c}}{2}+M_{f w}^{c},
\end{array}\right.
$$

where $q_{1} \approx \theta_{f}$ - a generalized coordinate corresponding to the angular displacement around the longitudinal axis of the railway ferry; $q_{2} \approx \theta_{f w}$ - a generalized coordinate corresponding to the angular displacement around the longitudinal axis of the flat wagon; $q_{3} \approx \theta_{c}$ - a generalized coordinate corresponding to the angular displacement around the longitudinal axis of the container. The origin of the coordinate system is located at the center of the mass of the railway ferry.

For the railway ferry: $D$ - weight water extrusion ; $B$ - width; $h$ - height of the board; $\Lambda_{\theta}$ - coefficient of fluctuations resistance; $z_{g}$ - coordinate of the centre of gravity; $p_{r f}^{\prime}-$ wind load on the surface projection; $F(t)$ - the law of the action effort that interferes with the movement of a railway ferry with wagons located on its decks.

For flat wagon with containers: $I_{f w}^{\theta}-$ moment of inertia of the flat wagon; $h_{f w}$ - height of the lateral surface of the flat wagon; $p_{f w}^{\prime}$ - wind load on the lateral surface of the flat wagon; $M_{f w}^{D}$ - moment of forces occurring between the flat wagon and the deck of the railway ferry at angular displacements relative to the longitudinal axis; $M_{f w}^{C}-$ moment of forces occurring between the flat wagon and containers at angular displacements relative to the longitudinal axis; $I_{C}^{\theta}$ - moment of inertia of the container; $h_{C}$ - height of the side surface of 
the container; $p_{C}^{\prime}$ - wind load on the side of the container; $M_{C}^{f w}$ - moment of forces occurring between the container and the flat wagon at angular displacements relative to the longitudinal axis.

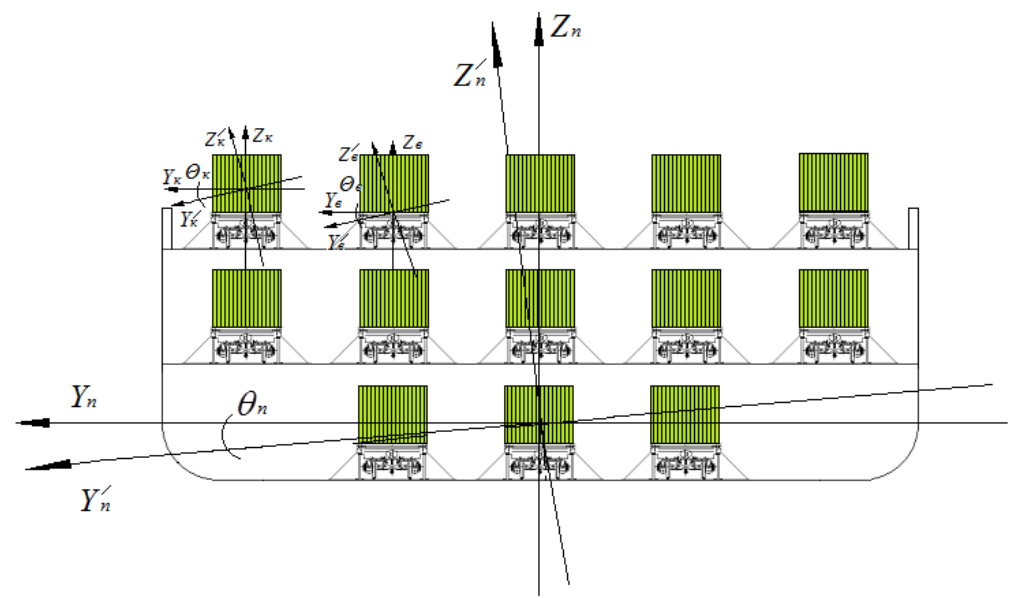

Fig. 4. Calculation scheme to research an angular displacement relative to the longitudinal axis of the railway ferry loaded with flat wagons with containers

The determination of the resistance coefficient by the fluctuation of the railway ferry is carried out in relatively to [13]:

$$
\Lambda_{\theta}=\omega_{0}^{\prime} \sqrt[8]{\frac{\left(I_{x}+\lambda\right) g}{D \cdot h^{2}}}\left(1+2 F r+10 F r^{2}\right),
$$

where $\lambda$ - connected moment of inertia of the mass of the fluid; $I_{x}$ - moment of inertia of the mass of the railway ferry relative to the longitudinal axis; $\mathrm{Fr}$ - is the Froude number, which depends on the speed of movement; $\omega_{o}^{\prime}$ - coefficient, which is determined by a special nomogram and depends on the ratio of $B / T$, where $T$ is the sediment of the railway ferry, $m$ [13].

When compiling the mathematical model, it is taken into account that the flat wagon has its own degree of freedom relative to the deck of the railway ferry, which may be due to:

- inequality of the deck (Fig. 5a),

- possible deviations in the geometry of the body (Fig. $5 b$ and $5 c$ ),

- unsymmetrical fixing of the flat wagon (Fig. 5d), etc.

Also, the model takes into account the possible movement of the container relative to the frame of the flat wagon at angular displacements relative to the longitudinal axis, which are conditioned by the presence of a gap in a pair of "fitting stop-fitting" [14-16]. In this case, the interaction of the flat wagon with the container was taken into account through the moment of the forces between the fitting stops and fittings. The percussive action of sea waves on the body of railway ferry with wagons placed on its board was not taken into account. The model takes into account the dissipative component that arises during the fluctuations of the railway ferry in the conditions of the sea shaking, which causes a resistance to its movement, and also the course angles of the sea wave in relation to the body of the railway ferry and wind load acting on the surface projection of the railway ferry, the flat wagon, located relative to the upper deck and containers. 


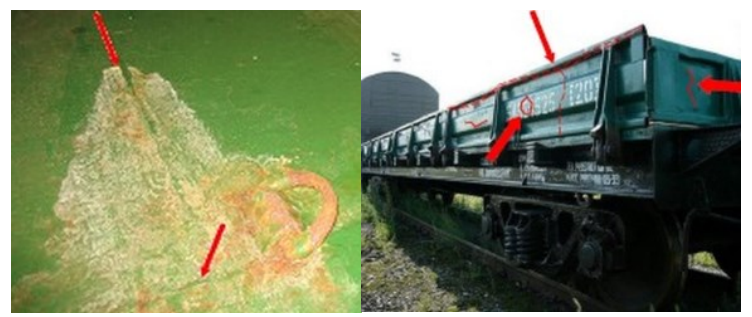

a)

b)

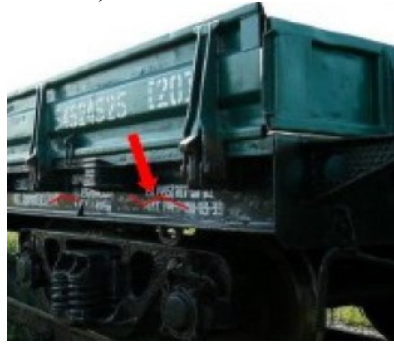

c)

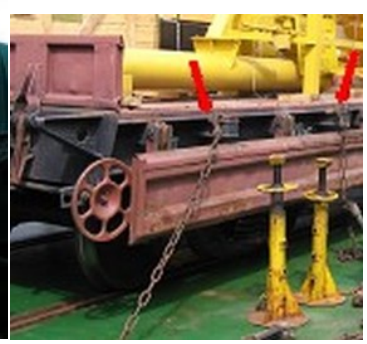

d)

Fig. 5. Factors which may cause the own degree of freedom of the bearing structure of the wagon relative to the deck of the railway ferry: a) uneven of the deck; b), c) deviation in the geometry of the body; d) asymmetry of fixing relative to the deck

When compiling the model, the trochoidal movement law of the perturbing action (sea wave) on the railway ferry with the bodies of wagons located on its decks was taken into account. The profile of such a wave is described by the equations (6):

$$
\begin{aligned}
& x=a+\operatorname{Re}^{k b} \sin (k a+\omega t), \\
& z=b-\operatorname{Re}^{k b} \cos (k a+\omega t),
\end{aligned}
$$

where $a$ and $b$ are the horizontal and vertical coordinates of the center of the trajectory, behind which the particle rotates, which has the coordinates $x$ and $z$ for the time being; $R$ - is the radius of the trajectory at which the particles are rotated; $\omega$ - frequency of the sea wave; $k$ - frequency of the trajectory of perturbing effort [17].

The input parameters of the model are the technical characteristics of the railway ferry, flat wagon, container, and hydrometeorological characteristics of the swimming water area. The calculations are carried out in relation to the railway ferry "Heroes of Shipka", which moves in the Black Sea water area. Hydrometeorological characteristics of the Black Sea waters are determined by the data given in [18].

In calculations, accepted the value of the radius of the wave trajectory $R=4 \mathrm{~m}$, the frequency of the trajectory of the perturbing effort $k=0.45$, the period $-9 \mathrm{~s}$, the frequency of the sea wave is determined taking into account its course angle $\chi$ in relation to the body of the railway ferry $\left(\chi=0^{\circ}-180^{\circ}\right)$.

In determining the moment of forces occurring between the flat wagon and the deck, the gross weight component is taken into account, with an allowance the friction force between the elements of the wagon structure (the axle-box knot in relation to the axle of the wheelset, the frame of the bogie relative to the axle-box knot, the movement of the body pivot bearing on the centre plate, etc. [19]).

In determining the force moment between the flat wagon and the container, the horizontal component of the gross weight of the container, the frictional forces between the fitting stop and fitting, and the geometry of the fitting stop are taken into account. 
The solution of differential equations was carried out using the Runge-Kutta method in the software environment of MathCad [20,21]. The results of calculations are shown in Fig. 5.

Based on the calculations it was established that the greatest values of acceleration arise at the course angles of the wave relative to the body of the railway ferry and, at the same values, the greatest frequency of acceleration can be traced (Fig. 6a).

Accelerations that act on the bearing structure of the flat wagon (Fig. 6b) have somewhat different characteristics than the railway ferry it is connected with the effect of friction forces that arise between the components of the wagon. The same applies to the container, the possible displacement of which is conditioned by the presence of a technological gap between the fitting stop and the fitting (Fig. 6c).The same applies to the container, the possible displacement of which is conditioned by the presence of a technological gap between the fitting stop and the fitting (Fig. 6c).That is, the carriage platform has its own degree of freedom, limited by the magnitude of possible displacements of the design elements, after which it will repeat the trajectory of moving the railway ferry. The container has its own degree of freedom by the time when the supporting of the vertical wall of the fitting in the fitting stop occurs.

At the same time, the maximum acceleration of the container was about $2.5 \mathrm{~m} / \mathrm{s}^{2}$, the flat wagon $-1.7 \mathrm{~m} / \mathrm{s}^{2}$, the railway ferry $-0.7 \mathrm{~m} / \mathrm{s}^{2}$. Numerical values of accelerations are given without taking into account the component of acceleration of free fall.

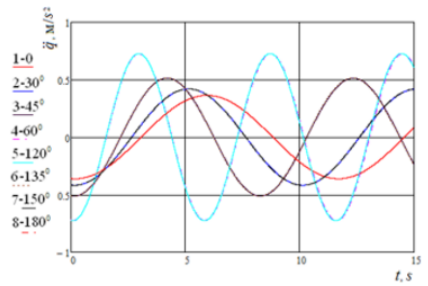

a)

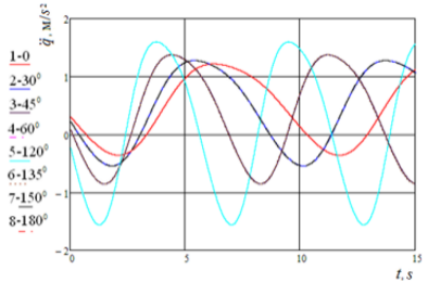

b)

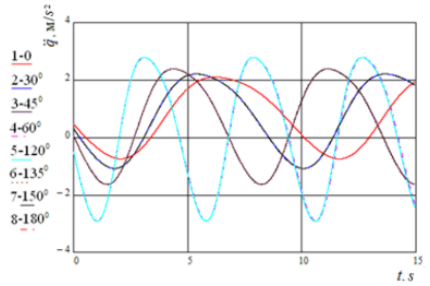

c)

Fig. 6. Acceleration of the railway ferry, loaded with flat wagons with containers: a) railway ferry; b) flat wagon; c) the container

The obtained acceleration values are used in drawing up the model of the structural strength of the bearing structure of the flat wagon of the articulated type, taking into account its transportation on the railway ferry (Fig. 7).

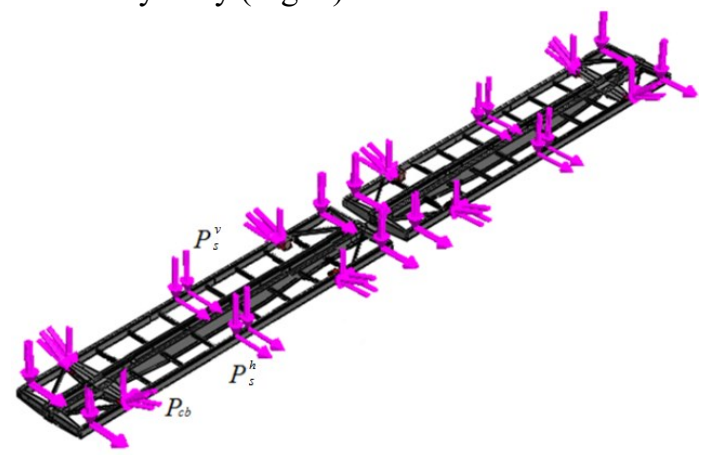

Fig. 7. Model of durability of advanced structural design of a flat wagon of an articulated type with nodes for fixing relative to the deck of a railway ferry

The fixing of the model was carried out in the zones of the supporting of the bearing structure and the bogies. For modeling the supporting of bearing structure on the working 
surfaces of the mechanical shutter-jacks on it were installed overlays of round shape, the diameter of which is equal to the diameter of the working part of the stop-jack (Fig. 8).

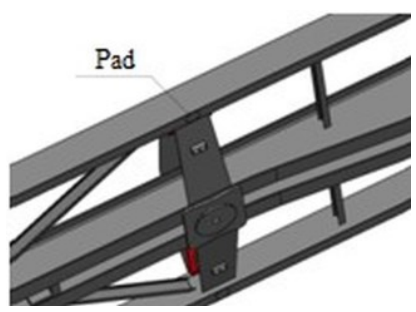

Fig. 8. Placement of the lining pad for modeling the supporting of the bearing structure of the flat wagon on the mechanical stop-jack

It is taken into account that on the bearing structure of the flat wagon acts the vertical load in the zones of supporting the containers on the fitting stop $P_{s}^{v}$, horizontal, which acts on the fitting stop of the flat wagon from the fitting of the container at its angular displacements relative to the longitudinal axis $P_{s}^{h}$ (Fig. 9a), and the efforts from the chain binders on the knots for fixing relative to the deck $P_{c b}$ (Fig. 9b). At the same time $P_{c b}^{x} \approx 30 \mathrm{kN} ; P_{c b}^{y} \approx 40 \mathrm{kN} ; P_{c b}^{z} \approx 30 \mathrm{kN}$.

The calculation is based on the finite element method in the CosmosWorks software environment. The graph-analytical method was used to determine the optimal number of grid elements. The number of nodes in the grid was 148723 , elements -462131 . The maximum size of the element is $200 \mathrm{~mm}$, the minimum $-40 \mathrm{~mm}$. The minimum number of elements in the circle was 9 , the ratio of increasing the size of elements in the grid $-1,7$. The maximum aspect ratio is 6182.6 , the percentage of elements with side ratios of less than 3-13.4, more than 10-33.7.

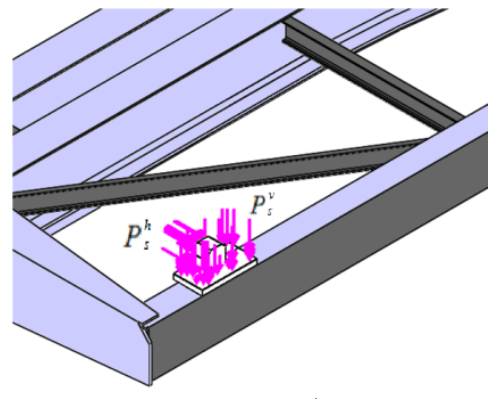

a)

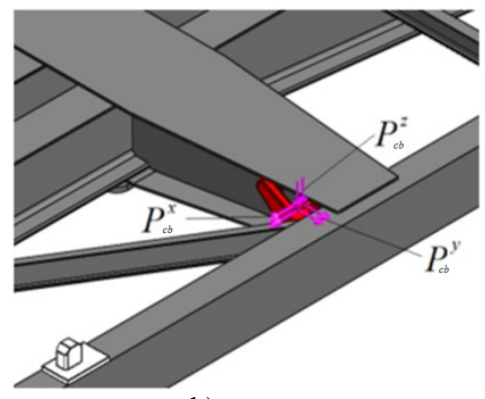

b)

Fig. 9. Scheme of applying the load to the elements of the bearing structure of a flat wagon of an articulated type during transportation on a railway ferry: a) to the fitting stop of the flat wagon from the container; b) to the knot for fixing of chain binders

The results of calculating the bearing structure of the flat wagon are shown in Fig. 10-13. From the conducted researches it can be concluded that the maximum equivalent tenses are concentrated in the interaction zone of the bolster beam with the main longitudinal and make up about $300 \mathrm{MPa}$, that is, they do not exceed the permissible for the given steel grades of metal structure [22], the maximum displacements arise in the middle part the main longitudinal beams of the frame and equal $15.6 \mathrm{~mm}$, the maximum deformation $-1.52 \cdot 10^{-2}$. 


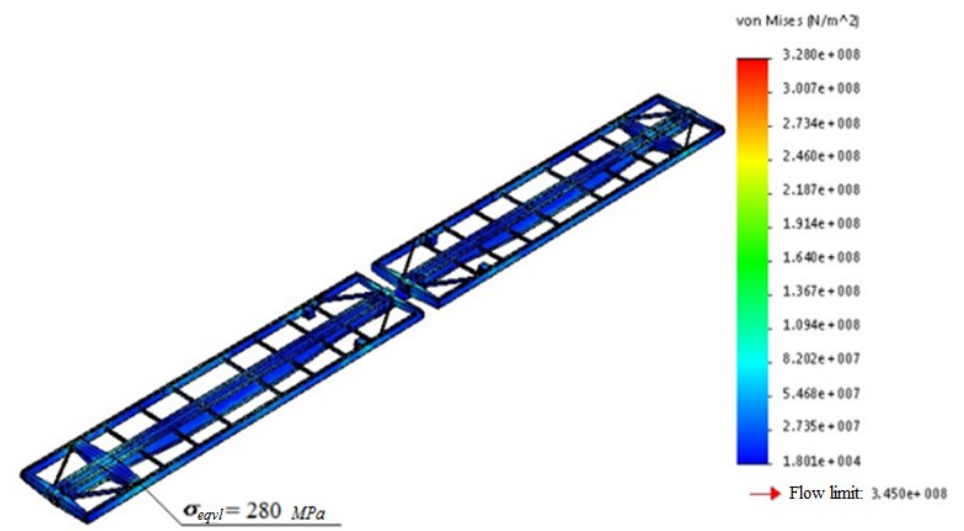

Fig. 10. The tense state of the improved bearing structure of a flat wagon of an articulated type during transportation on a railway ferry

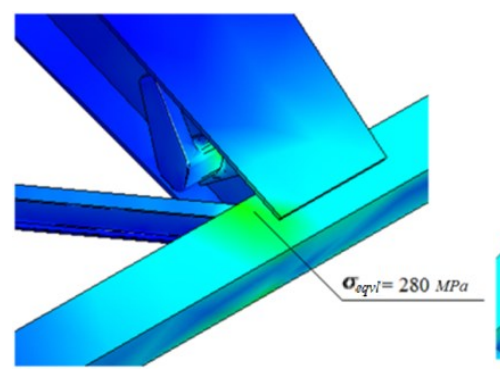

a)

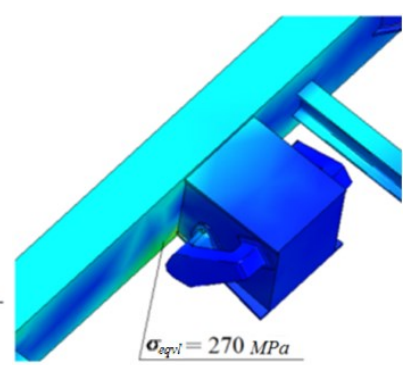

b)

Fig. 11. The tense state of the main longitudinal beam of the bearing structure of the flat wagon in the locations of the nodes for fixing the chain binders a) in the zone of interaction with the bolster beam; b) in the zone of interaction with the superstructure for placing the fixing node of the chain binder

The bearing structure of the flat wagon, taking into account measures for its improvement, is designed for fatigue strength in the CosmosWorks software environment. The test base had $10^{7}$ cycles. The fatigue curve for the study of fatigue strength is obtained according to the data on the fatigue strength of the steel grade 09G2C. The results of the calculation allowed to conclude that the fatigue strength of the bearing structure of the flat wagon is ensured.

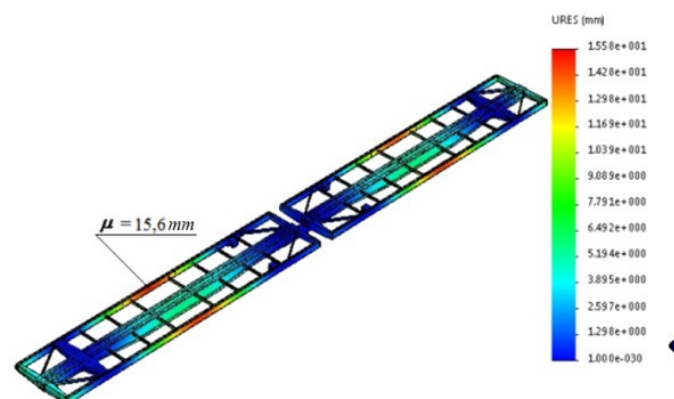

Fig. 12. Moving in the knots of the improved bearing structure of a flat wagon of an articulated type during transportation on a railway ferry

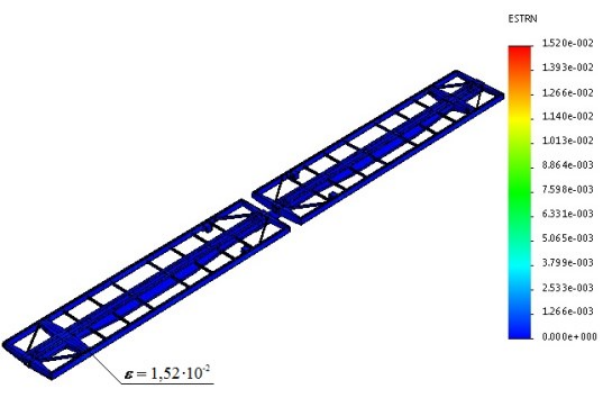

Fig. 13. Deformations in the improved bearing structure of a flat wagon of an articulated type during transportation on a railway ferry 


\section{Conclusions}

Based on the research carried out, the following conclusions can be drawn:

- in order to increase the efficiency of combined transportation, it is proposed to manufacture articulated flat wagons based on typical constructions,

- in order to ensure the strength of the bearing structures of the flat wagons of the articulated type at the stage of their design and manufacturing, it is necessary to take into account the adjusted values of the dynamic loads that can act on them not only during operation on main lines, but also during transportation on railway ferries,

- for reliable fixing of the bearing structure of the flat wagon on the deck of the railway ferry, it is necessary to provide knots on it for interaction with the chain binders, which must withstand the load from it to $196.2 \mathrm{kN}$, also to have a margin of construction design equal to - the design resistance the welding seam at the site of the interaction of the fixing knot with the bearing structure of the flat wagon should be $R_{Y}^{C R}=196 \mathrm{MPa}$,

- when calculating the strength of the bearing structure of the flat wagon attention must be paid to the effort that will be transmitted to it through the chain binders when fluctuations in the railroad ferry. Accelerations acting on the bearing structure of a flat wagon at angular movements relative to the longitudinal axis, regardless of the gross weight component should be taken at about $1.7 \mathrm{~m} / \mathrm{s}$, and for the container - about $2.5 \mathrm{~m} / \mathrm{s}$,

- the conducted researches will promote increase of efficiency of combined transport in the direction of international transport corridors.

\section{References}

1. G. Divya Priya, A. Swarnakumari, Modeling and analysis of twenty tonne heavy duty trolley. B Intern. J. of Innovative Technology and Research, 2, 6, 1568-1580 (2014)

2. W. Krason, T. Niezgoda, Fe numerical tests of railway wagon for intermodal transport according to PN-EU standards. Bulletin of the Polish Academy of Sciences Technical Sciences, 62, 4, 843-851 (2014)

3. N. Lysikov, R. Kovalev, G. Mikheev, Stress load and durability analysis of railway vehicles using multibody approach. Transport problems - Problemy Transportu, 2, 3, 49-56 (2007)

4. N. Lysikov, R. Kovalev, G. Mikheev, Stress load and durability analysis of railway vehicles using multibody approach. Transport problems - Problemy Transportu, 2, 3, 49-56 (2007)

5. WBN Waggonbau Niesky GmbH: Developing a flexible platform of freight wagons. Intern. Edition, 1, 46 (2016)

6. O.V. Fomin, Increase of the Freight Wagons's Ideality Degree and Prognostication of Their Evolution Stages. Scientific Bulletin of the NMU, 2, 68-76 (2015)

7. A.O. Lovska, A.V. Rybin, Research of dynamic loading of a car-platform during maneuveringco-operators. East-Europe. journ advanced technology, 3, 7 (81), 4-8 (2016)

8. N. L. Gurzhy, Improvement of the technical characteristics of a sectional wagonplatform by improving the design. author's abstract. dis.cand. of tech. Sciences: Dnipropetrovsk National University of Railway Transport named after Academician V. Lazaryan.-Dnepropetrovsk, (2010)

9. Lovska Alyona, Simulation of loads on the carrying structure of an articulated flat car in combined transportation. International Journal of Engineering \& Technology, 7, (4.3), $140-146$ (2018). 
10. R. I. Vysnyak, A. O. Lovskaya, E. M. Keba, Development of measures to ensure the preservation and adaptation of structures of wagons to their reliable interaction with multi-winding devices for fixing relative to decks of railway ferries. Prob. sciences works. Donetsk: DonIZT, 21, 110 - 119 (2010)

11. Instruction for general cargoes fixing during sea transportation for ferry "Heroes of Shipka". Cargo Securing Manual for ferry "Heroes of Shipka" No. 2512. 02. Officer. ed. Odessa: Min. transport of Ukraine. State Department of sea and river transport. P.51 (1997)

12. Alyamovskiy A. A. SolidWorks/COSMOSWorks 2006 - 2007. Engineering analysis by the finite element method, Moscow, 784, (2007)

13. S.N. Blagoveshchensky, A.N. Refrigerated, Handbook on static and dynamics of the ship. In two volumes. Izd. 2nd ed. and add Vol.2. Dynamics (duck) of the ship "Shipbuilding", 176 (1975)

14. Containers are universal. Types, basic parameters and dimensions. GOST 18477-79. [Valid from 11.2004] - M.: IPC standards, 11 (2004)

15. Containers are universal. General specifications. GOST 20259-80. - [Valid from 07.2002]. M.: IPC standards, 17, (2002)

16. Fittings for angular large containers. Design and dimensions. GOST 20527-82. [Effective from October 26, 2004]. M.: IPC standards, 9 (2004)

17. V.V. Lugovskii, Dynamics of the sea. Selected questions connected with the doctrine of ship's seaworthiness: [Textbook for studios in the specialty "Hydroaerodynamics", 199 (1976)

18. Wind and waves in the oceans and seas. background data [under. Ed. I.N. Davydana], 360 (1974)

19. V.V. Lukin, L.A. Shadur, V.I. Koturunov and other, Design and calculation of wagons. UMK of the Ministry of Internal Affairs of Russia, 731 (2000)

20. V. Dyakonov,M ATHCAD 8/2000: special reference book. St. Petersburg: Peter, 592 (2000)

21. D.V. Kiryanov, Mathcad 13. SPb: BHV, 608 (2006)

22. Railway applications - Structural requirements of railway vehicle bodies - Part 2: Freight wagons. EN 12663-2 